\title{
MORE ON COMPLEMENTS OF MINIMAL SPANNING SURFACES
}

\author{
R. J. DAIGLE ${ }^{1}$
}

\begin{abstract}
W. R. Alford in volume 91 of the Annals of Mathematics has shown the existence of a knot which has two minimal spanning surfaces whose complements in $S^{3}$ are not homeomorphic. The trefoil knot is a companion to the knot. This paper shows that any nontrivial knot $k$ is a companion to a knot $K$ which has at least two minimal spanning surfaces.
\end{abstract}

Introduction. In [1], W. R. Alford exhibited a knot $k$ and two minimal spanning surfaces $S_{1}$ and $S_{2}$ for $k$ such that $S^{3}-S_{i}$ are not homeomorphic. The knot was formed by sending the torus $T$ containing the knot $l$ in Fig. 1 faithfully to a regular neighborhood of the trefoil knot.

In a later paper [2], Alford and C. B. Schaufele constructed knots with $2^{m}$ really distinct minimal spanning surfaces; the surfaces do not have homeomorphic complements. The examples were constructed by sending the torus $T$ containing the knot $l$ in Fig. 1 faithfully to a regular neighborhood of the sum of $m$ "nice" knots. The selection of the knots was strongly influenced by their algebraic properties.

The purpose of this paper is to show that any nontrivial knot is a companion to a knot $K$ which has at least two minimal surfaces.

The knot $K$ is the image of the knot $l$ in $T$ in Fig. 1 under a faithful homeomorphism of the solid torus $T$ to a regular neighborhood $V$ of the knot $l$.

The Alexander polynomial of $K$ is $(2-t) \cdot(2 t-1)$ [4] for any nontrivial $k$ used. Thus $K$ had genus at least one. The spanning surfaces for $K$ have genus one, so $K$ has genus one.

The surfaces. The surfaces for $K$ are constructed as in [2]. The knot $l$ is spanned by a singular disk in $T$ as shown in Fig. 2.

Only one side is shown; the singularities are in heavy lines.

Received by the editors June 4, 1971 and, in revised form, November 8, 1971.

AMS (MOS) subject classifications (1970). Primary 55A25; Secondary 57A10.

Key words and phrases. Knot, companion, knot group, minimal spanning surface of a knot, spine of a surface.

${ }^{1}$ The contents of this paper are part of the author's doctoral dissertation at the University of Georgia under the direction of W. R. Alford. This research was supported by NDEA Title IV Fellowship 67-02845-1. 
The image of the disk in $V$ is as in Fig. 3 with the band portion tied in the knot $k$ (with twists in the band).

The two singularities are cut out and a tube is attached to the boundaries of the excised disks as indicated in Fig. 4.

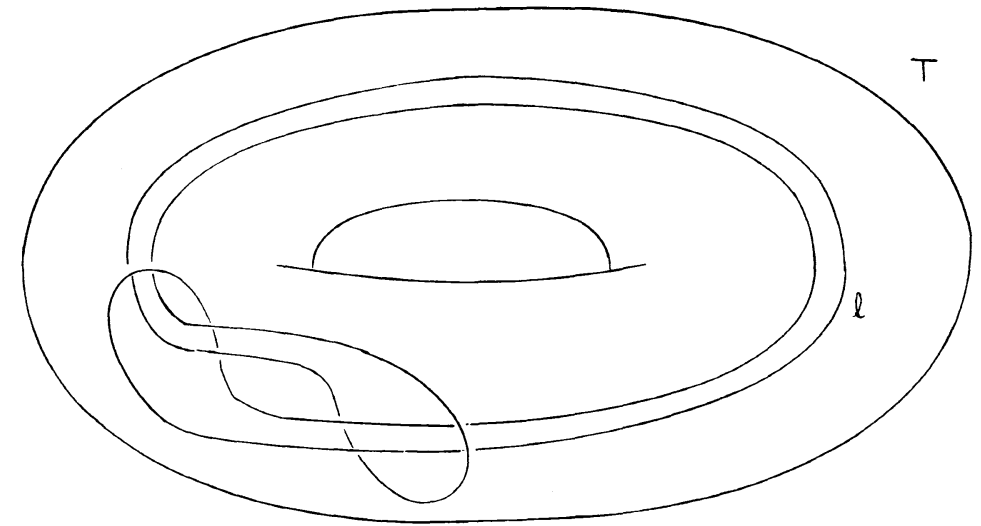

Figure 1

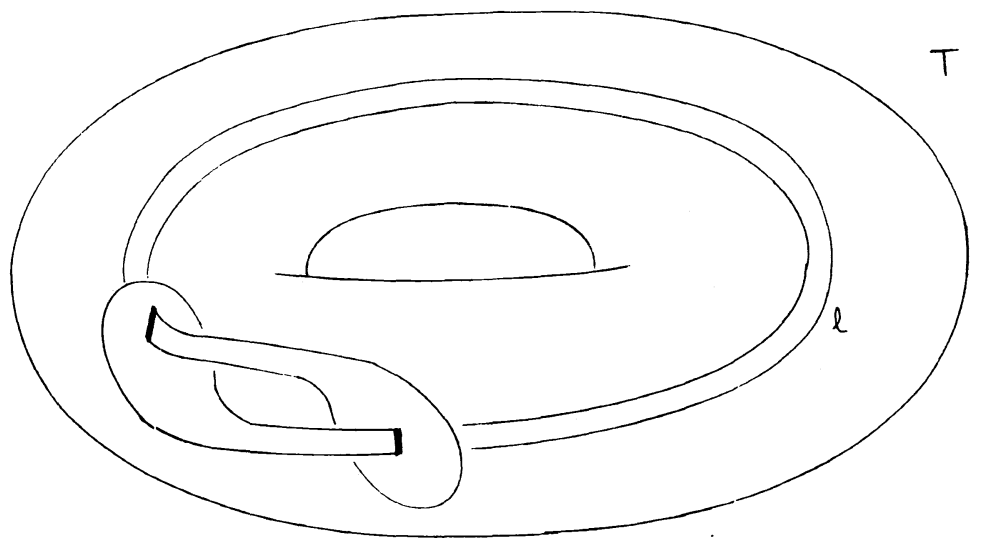

Figure 2

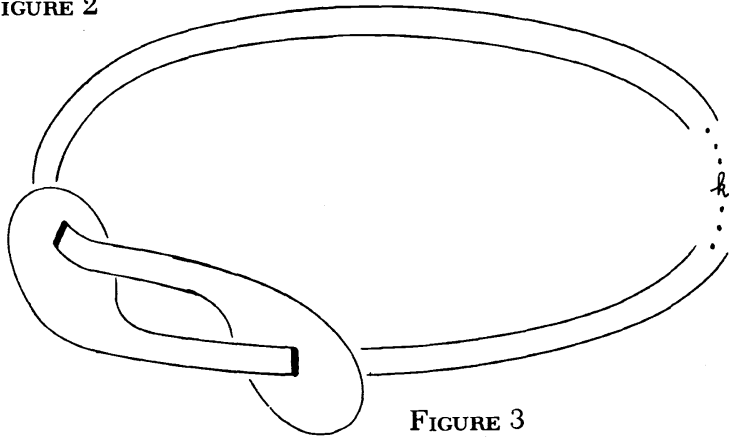


There are two possibilities for the tube surrounding the knot $k$ in the band as indicated in Figs. 5 and 6 for the figure eight knot.

$S_{1}$ will be the surface when the tube does not go "through" the knotted band; $S_{2}$ when the tube does go "through" the knotted band. A spine for $S_{1}$ is shown in Fig. 7. Fig. 8 has the same knot type as Fig. 7. Thus $\pi_{1}\left(S^{3}-S_{1}\right)$ is the free product of the integers with the knot group of $k$.

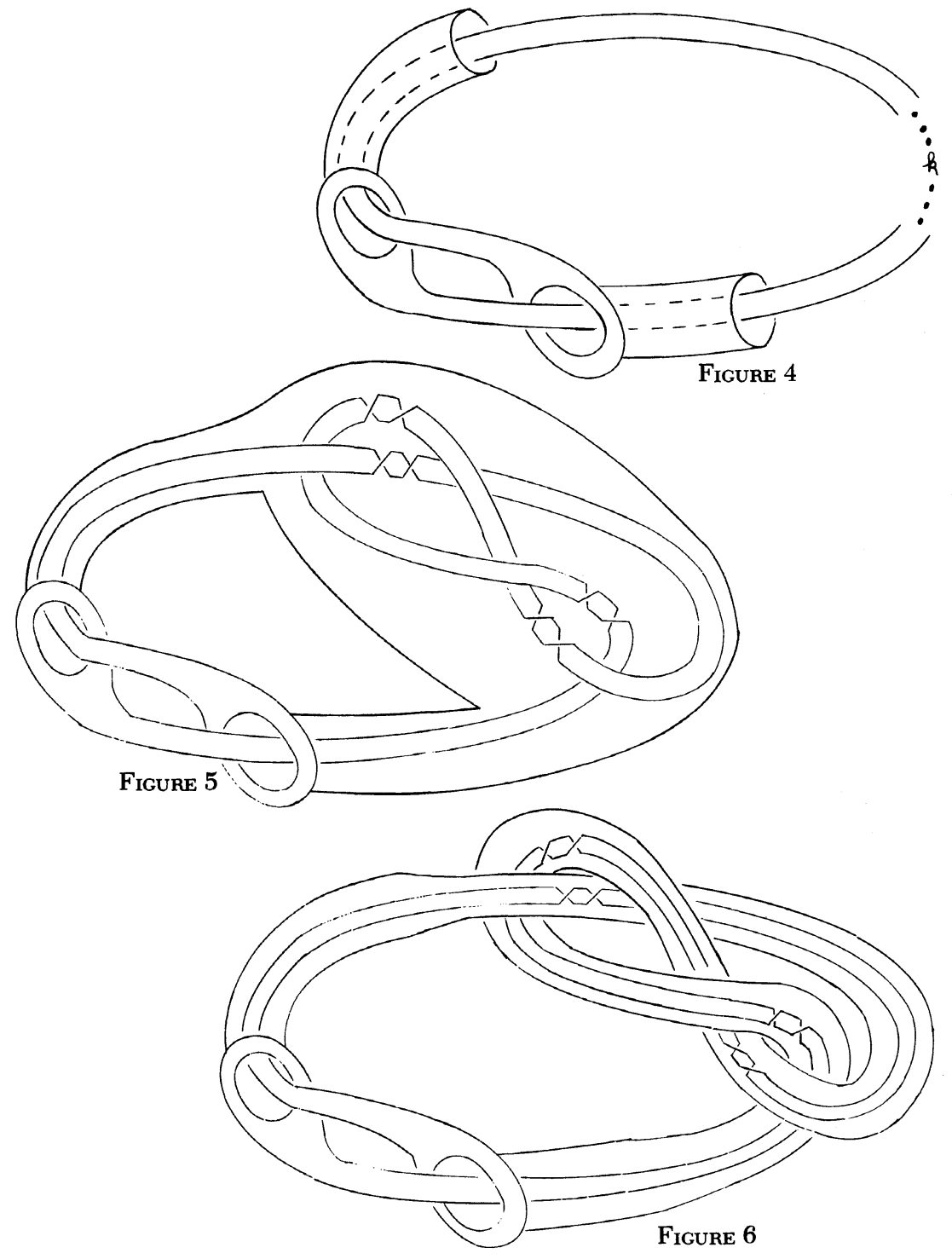




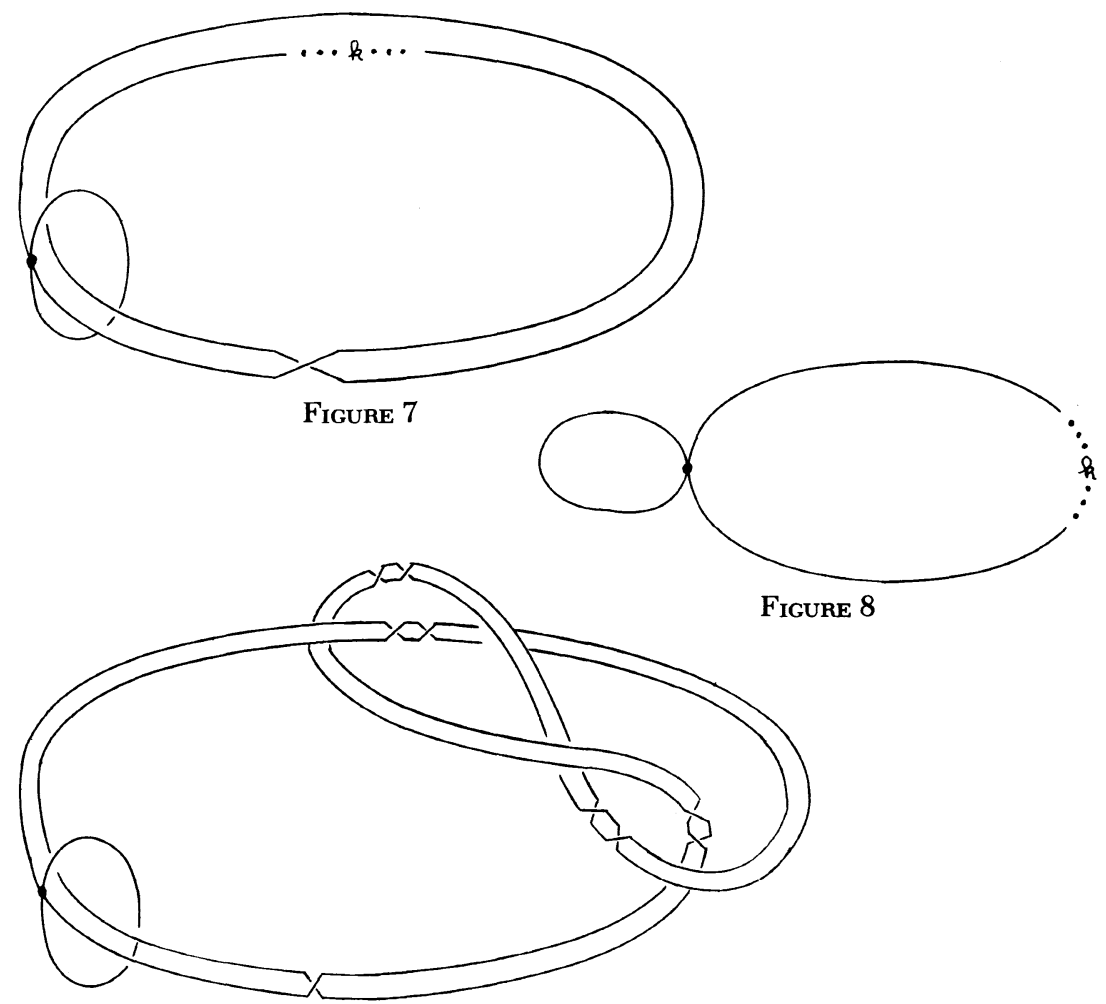

Figure 9

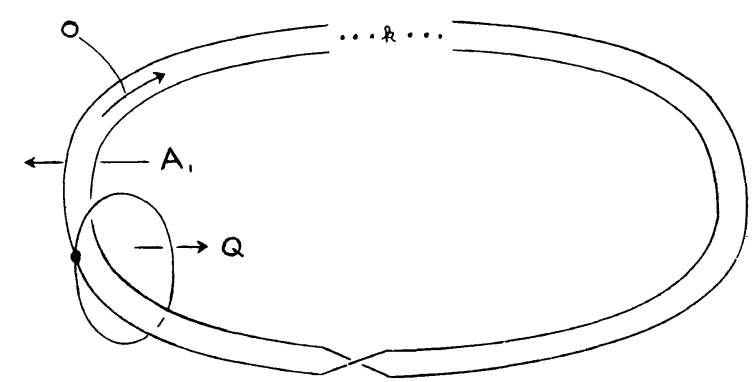

Figure 10

A spine $C$ for $S_{2}$ will be taken so that the part of the spine which lies on the tube is "homologous" to zero in the complement of the knot $k$ in the band. An example is shown in Fig. 9.

Let $G=\left(A_{1}, \cdots, A_{n}: R_{1}, \cdots, R_{n}\right)$ be the group of $k$ obtained from an over presentation with $A_{1}$ as in Fig. 10. 
Let $C_{1}$ be the part of the spine $C$ bounding a disk in $S^{3}$ and $C_{2}$ the part bounding a Möbius band $M$. Let $T$ be the boundary of a relative regular neighborhood of $M$ in the complement of $C_{1}-C_{1} \cap C_{2}$. Let $O$ be the center line of $M$. If $S^{3}-C$ is decomposed into the part not inside $T$ and the part not outside $T$, then a simple application of Van Kampen's Theorem [3] gives $\pi_{1}\left(S^{3}-S_{2}\right)=\left(O, Q, A_{1}, \cdots, A_{n}: R_{1}, \cdots, R_{n}, O^{2}=Q W A^{-1} Q W\right)$ where $W$ is the word in $G$ obtained from twisting the spine on the tube around the band. $W$ is a generator of the first homology of the boundary of a small regular neighborhood of $k$ in $S^{3}$.

Let $G_{1}=\pi_{1}\left(S^{3}-S_{1}\right)=Z * H$ and $G_{2}=\pi_{1}\left(S^{3}-S_{2}\right)$ where $H$ is isomorphic to $G$. The theorem that will be proved is

THEOREM 1. $G_{1}$ and $G_{2}$ are not isomorphic.

Preliminaries. Suppose $\varphi: G_{2} \rightarrow G_{1}$ is an isomorphism. $G_{2}$ contains a copy of $G$, the knot group of $k . G$ is not a free product since $k$ is not trivial [8]. Therefore, since $\operatorname{rank}(G)>1, \varphi(G)$ is conjugate to a subgroup of the free factor $H$ in $G_{1}$ by the Kurosh Subgroup Theorem [5]. It can then be assumed that the isomorphism $\varphi$ also sends $G$ to a subgroup of $H$.

Let $z$ be a generator of $Z$ and let $\varphi(O)=v, \varphi(Q)=u, \varphi\left(A_{1}\right)=x$, $\varphi\left(W A_{1}^{-1}\right)=t, \varphi(W)=t^{\prime}=t \cdot x . \quad x, t$, and $t^{\prime}$ are in $H$. The following lemma will be needed later.

Lemma 2. $v, u^{2}, x, v^{2}, v^{2} x^{-1}, t, t^{\prime}, t^{\prime} \cdot t^{-1}$ are each nontrivial words in $G_{1}$.

Proof of Lemma 2. The first five are nontrivial because $G_{2}$ is a free product with amalgamation containing as a subgroup the free group generated by $Q$ free product with $G$. $A_{1}$ and $W$ generate $Z \oplus Z$ [7, p. 57] as a subgroup of $G$ since $k$ is nontrivial. Because $\varphi$ is an isomorphism, $t, t^{\prime}$ and $t^{\prime} t^{-1}$ cannot be trivial.

The relation $O^{2}=Q W A_{1}{ }^{-1} Q W$ in $G_{2}$ gives $v^{2}=u t u t^{\prime}$ in $G_{1}$. The strategy will be to show there is no $u \neq 1$ which satisfies the relation.

$v$ has one of the following as its reduced form ( $b_{i}$ 's belong to H):

Form 1: $v=b_{1} z^{\alpha(1)} \cdots b_{n} z^{\alpha(n)}$.

Form 2: $v=b_{1} z^{\alpha(1)} \cdots z^{\alpha(n-1)} b_{n}$.

Form 3: $v=z^{\alpha(1)} b_{1} \cdots z^{\alpha(n)} b_{n}$.

Form 4: $v=z^{\alpha(1)} b_{1} \cdots b_{n-1} z^{\alpha(n)}$.

Conjugation by an element of $H$ in $Z * H$ sends $H$ to itself. Thus conjugating Form 3 by $b_{n}^{-1}$ and Form 4 by $x$ gives rise 
to new isomorphisms of $G_{2}$ to $G_{1}$, sending $G$ to a subgroup of $H$ and giving the new $v$ 's Form 1 and Form 2 respectively. Form 2 can be changed to Form 1 when $b_{n} \cdot b_{1} \neq 1$. Thus the existence of $\varphi$ depends on $v$ 's ability to assume Form 1 or Form 2 with $b_{n} \cdot b_{1}=1$.

There are three cases to consider according to the reduced form of $v^{2}$.

Case 1. $v$ has Form $1, v^{2}=b_{1} z^{\alpha(1)} \cdots b_{n} z^{\alpha(n)} b_{1} z^{\alpha(1)} \cdots b_{n} z^{\alpha(n)}$ is already reduced.

Case 2. $v$ has Form 2 with $b_{n} \cdot b_{1}=1, v^{2}$ has reduced form $v^{2}=b_{1} z^{\alpha(1)} \cdots b_{q-1} z^{\alpha(q-1)}\left(b_{q} \cdot b_{n-q+1}\right) z^{\alpha(n-q+1)} \cdots z^{\alpha(n-1)} b_{n} \quad$ for $1 \leqq q<n$.

Case 3. $v$ has form 2 with $b_{n} \cdot b_{1}=1, v^{2}$ has reduced form $v^{2}=b_{1} z^{\alpha(1)} \cdots b_{q}\left(z^{\alpha(q)+\alpha(n-q)}\right) b_{n-q+1} \cdots z^{\alpha(n-1)} b_{n}$ for $1 \leqq q<n$.

Therefore to prove Theorem 1 , it need only be shown that Cases 1-3 cannot occur.

Proof of Theorem 1. The following lemma will contribute greatly to the demise of Case 1 and Case 2 .

Lemмa 3. Let $g_{i}$ 's be elements of $H$ and let $\beta(i)$ 's be integers. Suppose the following two lists of equations hold for integers $r, k$, and $p$ with $1 \leqq k-p \leqq p-1$ :

$$
\begin{array}{r} 
\begin{cases}g_{k-r+1} \cdot g_{r}=1 & \text { for } 2 \leqq r \leqq k-p, \\
\beta(k-r)+\beta(r)=0 & \text { for } 1 \leqq r \leqq k-p,\end{cases} \\
\begin{cases}g_{r}=g_{k-p+r} & \text { for } 2 \leqq r \leqq p-1, \\
\beta(r)=\beta(k-p+r) & \text { for } 1 \leqq r \leqq p-1 .\end{cases}
\end{array}
$$

Then either there is an $r, 2 \leqq r \leqq k-p$, so that $g_{r}=1$ or there is an $r, 1 \leqq r \leqq k-p$, so that $\beta(r)=0$.

Proof of Lemma 3. The differences $A=\{k-2 r+1: 2 \leqq r \leqq$ $k-p\}$ and $B=\{k-2 r: 1 \leqq r \leqq k-p\}$ of indices in (3.1) give $2(k-p)-1$ consecutive integers and hence all equivalence classes modulo $(k-p)$ since $k-p \geqq 1$. If $0 \bmod (k-p)$ appears in $A$ then there is an $r, 2 \leqq r \leqq k-p$ so that $g_{k-r+1} \cdot g_{r}=1$ and $(k-2+1) \equiv 0 \bmod (k-p)$. Using the latter fact and $k-r \leqq p-1$, one can deduce from (3.2) that $g_{r}=g_{k-r+1}$. Thus $g_{r}{ }^{2}=1$. $H$ has no torsion so $g_{r}=1$. The alternate conclusion is reached in a similar manner if $0 \bmod (k-p)$ appears in $B$.

Case 1. Note length $\left(v^{2}\right)=4 n>0, v^{2}$ begins with $b_{1} \neq 1$ from $H$ and ends with $z^{\alpha(n)} \neq 1$.

A. If $l(u)=2 k \geqq 2$ then either 


$$
u=a_{1} z^{\epsilon(1)} \cdots a_{k} z^{\epsilon(k)} \quad \text { or } \quad u=z^{\epsilon(1)} a_{1} \cdots z^{\epsilon(k)} a_{k} .
$$

If $u=a_{1} z^{\epsilon(1)} \cdots a_{k} z^{\epsilon(k)}$ then

$$
v^{2}=a_{1} z^{\epsilon(1)} \cdots a_{k} z^{\epsilon(k)}\left(t a_{1}\right) z^{\epsilon(1)} \cdots a_{k} z^{\epsilon(k)} t^{\prime} .
$$

Spelling forces cancellation. Because of length, $v^{2}=1$ is the only possibility, contradicting Lemma 2.

If $u=z^{\epsilon(1)} a_{1} \cdots z^{\epsilon(k)} a_{k}$ then

$$
v^{2}=z^{\epsilon(1)} a_{1} \cdots z^{\epsilon(k)}\left(a_{k} t\right) z^{\epsilon(1)} a_{1} \cdots z^{\epsilon(k)}\left(a_{k} t^{\prime}\right) .
$$

Because of spelling, $a_{k} t^{\prime}=1$ and $a_{k} t=1$. Hence $t=t^{\prime}$, a contradiction to Lemma 2.

B. If $l(u)=2 k-1 \geqq 1$ then either

$$
u=z^{\epsilon(1)} a_{1} \cdots a_{k-1} z^{\epsilon(k)} \quad \text { or } \quad u=a_{1} z^{\epsilon(1)} \cdots z^{\epsilon(k-1)} a_{k} .
$$

The second is the only possible choice because no cancellation is possible in computing $v^{2}$ by $v^{2}=u t u t^{\prime}$ and there is a contradiction because of spelling.

If $u=a_{1} z^{\epsilon(1)} \cdots z^{\epsilon(k-1)} a_{k}$ then

$$
v^{2}=a_{1} z^{\epsilon(1)} \cdots z^{\epsilon(k-1)}\left(a_{k} t a_{1}\right) z^{\epsilon(1)} \cdots z^{\epsilon(k-1)}\left(a_{k} t^{\prime}\right) .
$$

Length and spelling force $a_{k} t^{\prime}=1$ and

$$
v^{2}=a_{1} z^{\epsilon(1)} \cdots z^{\epsilon(k-1)}\left(a_{k} t a_{1}\right) z^{\epsilon(1)} \cdots z^{\epsilon(k-1)} .
$$

If $a_{k} t a_{1} \neq 1$ then using the two reduced forms for $v^{2}$ we have $a_{1}=b_{1}=a_{k} t a_{1}$ or $a_{k} t=1$. Since $a_{k} t^{\prime}=1$ already, we have a contradiction to Lemma 2. Thus $a_{k} t a_{1}=1$ and cancellation will continue until the reduced form is either

$$
v^{2}=a_{1} z^{\epsilon(1)} \cdots a_{p}\left(z^{\epsilon(p)+\epsilon(k-p)}\right) a_{k-p+1} \cdots a_{k-1} z^{\epsilon(k-1)}
$$

or

$$
v^{2}=a_{1} z^{\epsilon(1)} \cdots z^{\epsilon(p-1)}\left(a_{p} \cdot a_{k-p+1}\right) z^{\epsilon(k-p+1)} \cdots a_{k-1} z^{\epsilon(k-1)}
$$

for $1 \leqq p \leqq k-1$. The first can be eliminated because its length is $4 p-2$, which is not $0 \bmod 4$. If the reduced form for $v^{2}$ is the second then $l\left(v^{2}\right)=4(p-1)$, so $n=p-1$. Because of cancellations we have

$$
\begin{aligned}
a_{k-r+1} \cdot a_{r}=1 & \text { for } 2 \leqq r \leqq k-p, \\
\epsilon(k-r)+\epsilon(r)=0 & \text { for } 1 \leqq r \leqq k-p .
\end{aligned}
$$


Using the two reduced forms for $v^{2}$ we have

$$
\begin{aligned}
a_{r} & =a_{k-p+r} & & \text { for } 2 \leqq r \leqq p-1, \\
\epsilon(r) & =\epsilon(k-p+r) & & \text { for } 1 \leqq r \leqq p-1 .
\end{aligned}
$$

To apply Lemma 3 to obtain a contradiction that $u$ is reduced we need only show

LEMMA $4.1 \leqq k-p \leqq p-1$.

Proof of Lemma 4. $1 \leqq k-p$ follows from $1 \leqq p \leqq k-1$. If $k>2 p-1$ then in the cancellation to obtain the reduced form for $v^{2}$ using $v^{2}=u t u t^{\prime}$, the $k$ th letter of $u$ must be cancelled. The $k$ th letter of $u$ is $z^{\epsilon(k / 2)}$ if $k$ is even, $a_{(k+1) / 2}$ if $k$ is odd. Since the sum of the indices on the $\epsilon$ 's must be $k$ and on the $a$ 's must be $k+1$, either $2 \epsilon(k / 2)=0$ or $a_{(k+1) / 2}^{2}=1$. So either $\epsilon(k / 2)=0$ or $a_{(k+1) / 2}=1$, a contradiction to $u$ being reduced.

Lemma 3 can be applied to obtain that $u$ is not reduced, a contradiction. Thus $l(u) \neq 2 k-1 \geqq 1$.

This completes the proof that Case 1 cannot occur.

Case 2. We note that $l\left(u^{2}\right)=4 q-3>0 . v^{2}$ begins with $b_{1} \neq 1$ from $H, v^{2}$ ends with $b_{n} \neq 1$ from $H$. Because of cancellation to obtain the reduced form we have

$$
\begin{aligned}
b_{n-r+1} \cdot b_{r} & =1, & & 2 \leqq r \leqq n-q, \\
\alpha(n-r)+\alpha(r) & =0, & & 1 \leqq r \leqq n-q,
\end{aligned}
$$

half of the equations needed to apply Lemma 3.

Lemma 5. $1 \leqq n-q \leqq q-1$.

The proof is exactly as in Lemma 4 using $v$ instead of $u$.

A. If $l(u)=2 k \geqq 2$ then $u=z^{\epsilon(1)} a_{1} \cdots z^{\epsilon(k)} a_{k}$ or $u=a_{1} z^{\epsilon(1)} \cdots$ $a_{k} z^{\epsilon(k)}$.

If $u=z^{\epsilon(1)} a_{1} \cdots z^{\epsilon(k)} a_{k}$ then

$$
v^{2}=z^{\epsilon(1)} a_{1} \cdots z^{\epsilon(k)}\left(a_{k} t\right) z^{\epsilon(1)} a_{1} \cdots z^{\epsilon(k)}\left(a_{k} t^{\prime}\right) .
$$

Because of spelling, $z^{\epsilon(1)} a_{1} \cdots\left(a_{k} t\right) \cdots z^{\epsilon(k)}=1$; in particular, $a_{k} t=$ 1. Thus $v^{2}=a_{k} t^{\prime}=t^{-1} t^{\prime}=x$, a contradiction to Lemma 2 .

If $u=a_{1} z^{\epsilon(1)} \cdots a_{k} z^{\epsilon(k)}$ then

$$
v^{2}=a_{1} z^{\epsilon(1)} \cdots a_{k} z^{\epsilon(k)}\left(t a_{1}\right) z^{\epsilon(1)} \cdots a_{k} z^{\epsilon(k)} t^{\prime} .
$$

If $t a_{1} \neq 1$ then $v^{2}$ does not reduce further; $l\left(v^{2}\right)=4 k+1$, so $q=k+1$. Using the two reduced forms for $v^{2}$ we obtain 


$$
\begin{aligned}
b_{r} & =b_{n-q+r} & & \text { for } 2 \leqq r \leqq q-1, \\
\alpha(r) & =\alpha(n-q+r) & & \text { for } 1 \leqq r \leqq q-1 .
\end{aligned}
$$

Lemma 3 applied here gives a contradiction to $v$ being reduced. Thus $t a_{1}=1$.

Observe that $l\left(v^{2}\right)=1$ is impossible for then $t a_{1}=1$ and $v^{2}=a_{1} t^{\prime}$ imply $v^{2}=x$ which is impossible by Lemma 2. Thus if $v^{2}$ is allowed to reduce using $v^{2}=u t u t^{\prime}$ and if it is compared to the reduced form from $v$, we will always have the relation $a_{1}=b_{1}$ and $t^{\prime}=b_{n}$. Since $b_{n} \cdot b_{1}=1$ then $t^{\prime} \cdot a_{1}=1=t a_{1}$, a contradiction.

Hence $l(u) \neq 2 k \geqq 2$.

B. If $l(u)=2 k-1 \geqq 1$ then $u=z^{\epsilon(u)} a_{1} \cdots a_{k} z^{\epsilon(k)}$ or $u=a_{1} z^{\epsilon(1)}$ $\cdots z^{\epsilon(k-1)} a_{k}$.

The former cannot occur because no cancellation is possible for $v^{2}$ from $v^{2}=u t u t^{\prime}$ and $v^{2}$ is spelled incorrectly.

If $u=a_{1} z^{\epsilon(1)} \cdots z^{\epsilon(k-1)} a_{k}$ then

$$
v^{2}=a_{1} z^{\epsilon(1)} \cdots z^{\epsilon(k-1)}\left(a_{k} t a_{1}\right) z^{\epsilon(1)} \cdots a_{k-1} z^{\epsilon(k-1)}\left(a_{k} t^{\prime}\right) .
$$

If $a_{k} t^{\prime}=1$, spelling of $v^{2}$ forces $v^{2}=a_{1}$ and $a_{k} t a_{1}=1$. Since $t^{\prime}=t x$, $a_{1}=x$. Thus $v^{2}=x$ contradicting Lemma 2. Therefore $a_{k} t^{\prime} \neq 1$ and from this point the proof of the second part of $A$ of this case can be imitated to deduce that $u=a_{1} z^{\epsilon(1)} \cdots z^{\epsilon(k-1)} \cdots z^{\epsilon(k-1)} a_{k}$ is impossible. Hence $l(u) \neq 2 k-1 \geqq 1$.

This completes the proof that Case 2 cannot occur.

Case 3. We note that $l\left(v^{2}\right)=4 q-1>0, v^{2}$ begins with $b_{1} \neq 1$ from $H$ and ends with $b_{n} \neq 1$ from $H$.

A. If $l(u)=2 k \geqq 2$ then $u=z^{\epsilon(1)} a_{1} \cdots z^{\epsilon(k)} a_{k}$ or $u=a_{1} z^{\epsilon(1)} \cdots$ $a_{k} z^{z^{(k)}}$. The former is easily shown to be impossible by spelling and length arguments.

$$
\begin{aligned}
& \text { If } u=a_{1} z^{\epsilon(1)} \cdots a_{k} z^{\epsilon(k)} \text { then } \\
& \qquad v^{2}=a_{1} z^{\epsilon(1)} \cdots a_{k} z^{\epsilon(k)}\left(t a_{1}\right) z^{\epsilon(1)} \cdots a_{k} z^{\epsilon(k)} t^{\prime} .
\end{aligned}
$$

Because of length, $t a_{1}=1$ and since $l\left(v^{2}\right) \neq 1$ then using the two reduced forms for $v^{2}$ we always obtain the relations $a_{1}=b_{1}$ and $t^{\prime}=b_{n}$. Since $b_{n} \cdot b_{1}=1, t^{\prime} \cdot a_{1}=1=t a_{1}$, a contradiction. Thus $l(u) \neq 2 k \geqq 2$.

B. The proof that $l(u) \neq 2 k-1 \geqq 1$ is very much like part A of this case.

Thus Case 3 cannot occur and Theorem 1 is proved. 


\section{Bibliography}

1. W. R. Alford, Complements of minimal spanning surfaces of knots are not unique, Ann. of Math. (2) 91 (1970), 419-424. MR 40 \#6527.

2. W. R. Alford and C. B. Schaufele, Complements of minimal spanning surfaces are not unique. II, Topology of Manifolds, Markham, Chicago, Ill., 1970, pp. $87-96$.

3. R. H. Crowell and R. H. Fox, Introduction to knot theory, Ginn, Boston, Mass., 1963. MR 26 \#4348.

4. R. H. Fox, A quick trip through knot theory, Topology of 3-Manifolds and Related Topics (Proc. The Univ. of Georgia Inst., 1961), Prentice-Hall, Englewood Cliffs, N. J., 1962, pp. 120-167. MR 25 \#3522.

5. M. Hall, Jr., The theory of groups, Macmillan, New York, 1959. MR 21 \#1996.

6. A. G. Kuros, Theory of groups, GITTL, Moscow, 1956; English transl., Vol. II, Chelsea, New York, 1960. MR 18, 188; MR 22 \#727.

7. L. P. Neuwirth, Knot groups, Ann. of Math. Studies, no. 56, Princeton Univ. Press, Princeton, N. J., 1965. MR 31 \#734.

8. Ch. D. Papakyriakopoulos, On Dehn's lemma and the asphericity of knots, Ann. of Math. (2) 66 (1957), 1-26. MR 19, 761.

University of Georgia, Athens, Georgia 30601 